\title{
A TRANSVERSE RF DEFLECTING STRUCTURE FOR BUNCH LENGTH AND PHASE SPACE DIAGNOSTICS*
}

\author{
R. Akre, L. Bentson, P. Emma ${ }^{\S}$, P. Krejcik \\ Stanford Linear Accelerator Center, Stanford, CA 94309, USA
}

\begin{abstract}
A traveling wave transverse rf deflecting structure is discussed as a tool for bunch length measurement and as an aid to detailed phase space diagnostics in future FEL projects. The high frequency time variation of the deflecting fields is used to 'pitch' or 'yaw' the electron bunch where the resulting transverse beam width measured on a simple profile monitor represents a reliable, single-shot measure of the absolute bunch length. A small rf phase shift, from the zero-crossing, adds a net centroid kick to the bunch so that it intercepts an off-axis screen. The deflecting orf is then pulsed at a much lower rate than the machine rate, and a pulse-stealing process is used to monitor the bunch length continuously. Multiple screens at appropriate phase advance locations might also be used to monitor the 'slice' (i.e., time-correlated) transverse emittance. A screen placed at a point of significant momentum dispersion can also be used to reveal the time-dependent energy spread. Suitable S-band structures were built and tested in the 1960's at SLAC and one has now been installed in the SLAC linac and configured to measure bunch lengths of test beams. Simulations of these schemes are presented for the Linac Coherent Light Source [1] and the existing SLAC linac.
\end{abstract}

\section{INTRODUCTION}

One of the many technical challenges for future freeelectron lasers (FEL) and linear colliders is the measurement and diagnosis of their extremely short electron bunches. The rms bunch length in the Linac Coherent Light Source (LCLS) is $80 \mathrm{fsec}(24 \mu \mathrm{m})$ and that of the Next Linear Collider (NLC) [2] is $300 \mathrm{fs}$ $(90 \mu \mathrm{m})$. This is well beyond the range of streak cameras, but a similar concept can be applied by directly 'streaking' the electron beam using an rf deflecting field. This idea has been used in the past $[3,4]$ and has been suggested again recently [5]. The high frequency time variation of the deflecting fields is used to 'pitch' or 'yaw' the electron bunch, where the resulting transverse beam width measured on a simple profile monitor represents a reliable, single-shot measure of the absolute bunch length. Since the method effectively converts one of the profile monitor's transverse dimensions into time, the bunch can be analyzed in detail, revealing important time-correlations in the other phase space dimensions and

\footnotetext{
* Work supported by DOE contract DE-AC03-76SF00515.

$\S$ Emma@SLAC.Stanford.edu
}

allowing the 'time-sliced' rms dimensions of the beam to be measured. This can be an essential diagnostic in future FEL's where the time-sliced beam widths are of fundamental importance, and the long-term stability of these parameters needs continuous monitoring.

Suitable S-band traveling wave structures were built and tested in the 1960's at SLAC [6] and are still available today. These are 8-foot and 12-foot long structures capable of 25-30 MW of peak input power and up to $32 \mathrm{MV}$ of peak deflecting voltage. Such structures can be utilized for future machines and even as a basic diagnostic in the existing SLAC linac.

\section{THE DEFLECTING STRUCTURE}

The existing S-band deflecting structures are described in references [3] and [6]. The disk-loaded waveguide structure is fabricated from brazed stacks of machined copper cylinders with a period of $3.5 \mathrm{~cm}$. The iris radius is $2.24 \mathrm{~cm}$, which is almost twice that of the SLAC Sband accelerating structures. A cut-away view of the deflecting structure is shown in Figure 1. The polarization plane of the $\mathrm{TM}_{11}$ mode is determined by the orientation of the input coupler. Since minor imperfections in the structure could cause the mode to rotate, two additional holes are provided on either side of each iris to stabilize and prevent mode rotations.

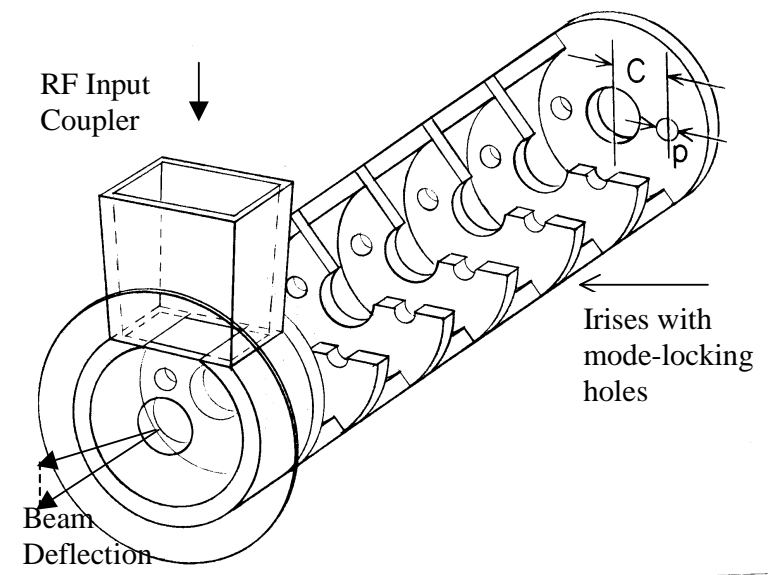

Figure 1. Schematic of a SLAC S-band transverse deflecting structure. The kick is vertical in this drawing.

The structure has a $2 \pi / 3$ phase shift per cell, as shown in Figure 2. Note that the (insignificant) longitudinal field is proportional to displacement from the axis and changes sign, passing through zero as the axis is crossed. 


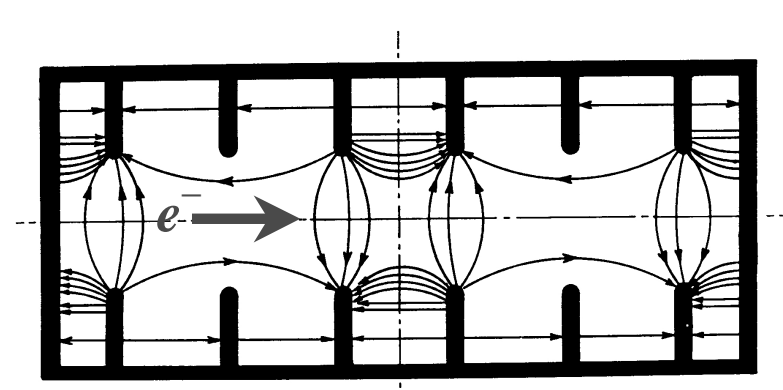

Figure 2. Time snap-shot of the field distribution of the $\mathrm{TM}_{11}$ mode with a $2 \pi / 3$ cell length.

At SLAC, there exists both 8-foot $(2.44 \mathrm{~m})$ and 12-foot $(3.64 \mathrm{~m})$ long structures, although we confine our consideration here to the 8 -foot structures. Measurements [7] and calculations [8] of the deflection versus power indicate a power to voltage relationship of

$$
V_{0} \approx\left(1.6 \mathrm{MV} / \mathrm{m} / \mathrm{MW}^{1 / 2}\right) L \sqrt{P_{0}},
$$

where $L$ is the structure length and $P_{0}$ is peak input power.

The aberration-free deflecting force imparts a transverse momentum on the bunch which varies in time over the passage of the bunch. The small kick angle, $\Delta x^{\prime}(<<1)$, as a function of longitudinal position along the bunch, $z$, is given by $(k \equiv 2 \pi / \lambda)$

$$
\Delta x^{\prime}(z)=\frac{e V_{0}}{p c} \sin (k z+\varphi) \approx \frac{e V_{0}}{p c}\left[\frac{2 \pi}{\lambda} z \cos \varphi+\sin \varphi\right],
$$

where $V_{0}$ is the peak voltage, $p$ is the beam's longitudinal momentum in the structure, and $\varphi$ is the rf phase $(=0$ at zero-crossing). The approximation is made that $|z|<<\lambda / 2 \pi$, so only the linear term in $z$ is retained.

From Eq. (2) it is clear (for $\langle z\rangle=0$ ) that a beam centroid deflection, $\left\langle\Delta x^{\prime}\right\rangle$, occurs for $\sin \varphi \neq 0$ and an angular 'yaw' (or 'pitch' for a vertical deflector) is generated along the bunch which is approximately linear in $z$. A spatial 'pitch' arises at a screen placed downstream of the deflector, where the beam has been transported through a transfer matrix with angular-to-spatial element $R_{12}=\left(\beta_{d} \beta_{s}\right)^{1 / 2} \sin \Delta \psi$. Here, $\beta_{d}$ is the beta function at the deflector, $\beta_{s}$ is the beta function at the screen, and $\Delta \psi$ is the betatron phase advance from deflector to screen. The transverse position of each ultra-relativistic electron on the screen is then given by

$$
\Delta x(z) \approx \frac{e V_{0}}{p c} \sqrt{\beta_{d} \beta_{s}} \sin \Delta \psi\left(\frac{2 \pi}{\lambda} z \cos \varphi+\sin \varphi\right) .
$$

Taking the mean value over the ensemble of particles, for the nominal case with $\langle z\rangle=0$, gives the transverse centroid offset at the screen.

$$
\langle\Delta x\rangle=\frac{e V_{0}}{p c} \sqrt{\beta_{d} \beta_{s}} \sin \Delta \psi \sin \varphi
$$

Similarly, the rms beam size on the screen is

$$
\left\langle(x-\langle x\rangle)^{2}\right\rangle^{1 / 2} \equiv \sigma_{x}=\sqrt{\sigma_{x_{0}}^{2}+\sigma_{z}^{2} \beta_{d} \beta_{s}\left(\frac{2 \pi e V_{0}}{\lambda p c} \sin \Delta \psi \cos \varphi\right)^{2}}
$$

where $\sigma_{x_{0}}$ is the nominal beam size on the screen (i.e., in the absence of a deflecting voltage), and $\sigma_{z}=\left\langle(z-\langle z\rangle)^{2}\right\rangle^{1 / 2}$ is the rms bunch length.

If the rf voltage is adequate, the beam size associated with the bunch length will dominate the nominal beam size, and the quadrature addition in Eq. (5) becomes a small correction. This requires the rf deflecting voltage be large compared to the scaled nominal beam size, or

$$
\left|e V_{0}\right| \gtrsim \frac{\lambda}{\pi \sigma_{z}} \frac{1}{\sin \Delta \psi \cos \varphi \mid} \sqrt{p c \cdot m c^{2} \frac{\varepsilon_{N}}{\beta_{d}}},
$$

which, when in equality, results in a quadrature correction of just $12.5 \%$. Here we have used, for the screen's nominal spot size,

$$
\sigma_{x_{0}}=\sqrt{\beta_{s} \varepsilon_{N} / \gamma},
$$

where $\gamma\left(=p c / m c^{2}\right)$ is the Lorentz energy factor, $m c^{2}$ is the electron rest energy, and $\varepsilon_{N}$ is the normalized transverse rms emittance (in the deflection plane). Eq. (6) indicates that the required voltage scales weakly with the squareroot of beam momentum and inversely with bunch length. The required voltage also decreases with smaller emittance and decreases for larger values of the beta function in the deflector. The beta function at the screen does not effect the 'signal-to-noise' relationship of Eq. (6), but it does scale the absolute measured spot size in Eq. (5). Similarly, a phase advance near $\Delta \psi=\pi / 2$, and an rf phase near zero-crossing are advantageous. Finally, a shorter rf wavelength also reduces the voltage required. For S-band $(\lambda \approx 10.5 \mathrm{~cm})$ and LCLS parameters with $p c \approx 6 \mathrm{GeV}, \quad \sigma_{z} \approx 22 \mu \mathrm{m}, \quad \varepsilon_{N} \approx 1 \mu \mathrm{m}, \quad \beta_{d} \approx 60 \mathrm{~m}$, the deflector voltage should be $\left|V_{0}\right| \geq 11 \mathrm{MV}$. For the SLAC linac parameters $\left(p c \approx 30 \mathrm{GeV}, \sigma_{z} \approx 600 \mu \mathrm{m}, \varepsilon_{N} \approx 5 \mu \mathrm{m}\right.$, $\beta_{d} \approx 50 \mathrm{~m}$ ), the deflector voltage only needs to be $\left|V_{0}\right| \geq 2.2 \mathrm{MV}$.

The possibility of acceleration between the deflecting structure and the screen should also be incorporated into these relationships. This is included by everywhere replacing the beta function product with

$$
\beta_{d} \beta_{s} \rightarrow \beta_{d} \beta_{s} E_{d} / E_{s},
$$

where $E_{d}(\approx p c)$ is the beam energy in the deflector and $E_{s}$ is the energy at the screen. Including acceleration, the measured bunch length is calculated using Eq (5).

$$
\sigma_{z}=\frac{\lambda}{2 \pi} \frac{\sqrt{E_{d} E_{s}}}{\left|e V_{0} \sin \Delta \psi \cos \varphi\right|} \sqrt{\frac{\left(\sigma_{x}^{2}-\sigma_{x_{0}}^{2}\right)}{\beta_{d} \beta_{s}}}
$$

The same deflecting structure can also be applied to the existing SLAC linac. With a bunch length of $600 \mu \mathrm{m}$ (in its shortest configuration) and a vertical emittance from the uncoupled damping ring of $5 \mu \mathrm{m}$, an S-band deflector can be very efficient. Table 1 lists some possible parameters for a 2.4-m $y$-deflector in the SLAC linac. 
Table 1. SLAC linac parameters (deflector at 29-4D) with uncoupled damping ring injector at 1-nC bunch charge.

\begin{tabular}{|l|c|c|r|}
\hline RF deflector voltage & $V_{0}$ & 10 & $\mathrm{MV}$ \\
Peak input power & $P_{0}$ & 6.5 & $\mathrm{MW}$ \\
RF deflector phase (crest: $90^{\circ}$ ) & $\varphi$ & 18 & $\mathrm{deg}$ \\
Nominal beam size & $\sigma_{x_{0}}$ & 68 & $\mu \mathrm{m}$ \\
Beam size with RF on (mean) & $\sigma_{x}$ & 575 & $\mu \mathrm{m}$ \\
Beta at deflector and screen & $\beta_{d}=\beta_{s}$ & 53 & $\mathrm{~m}$ \\
$\beta$-phase: deflector to screen & $\Delta \psi$ & 77 & $\mathrm{deg}$ \\
Normalized rms emittance, $y$ & $\varepsilon_{N}$ & 5 & $\mu \mathrm{m}$ \\
energy at deflector and screen & $E_{d}=E_{s}$ & 30 & $\mathrm{GeV}$ \\
RMS bunch length & $\sigma_{z}$ & 600 & $\mu \mathrm{m}$ \\
\hline
\end{tabular}

\section{SIMULATIONS AND TESTING}

Such a deflector has been located in linac-sector-29 at '29-4D' and a screen is already located just downstream at '29-902'. This is ideal, since no accelerator section was previously located at '29-4D', and a remotely moveable screen, and associated controls, already exist at '29-902'.

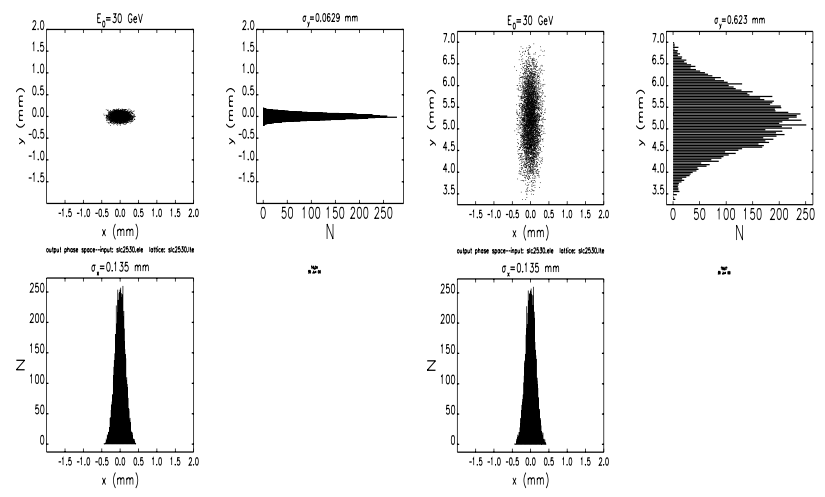

Figure 3. RF deflector OFF (left 3 plots), and ON (right 3 plots) for SLAC linac use at $30 \mathrm{GeV}, \sigma_{z} \approx 600 \mu \mathrm{m}, V_{0}=10 \mathrm{MV}$.

The simulated change in vertical beam profiles on screen '29-902' with cavity off (left) and on (right) in Figure 3 shows the simulation for this layout with a $10 \mathrm{MV}, \varphi \approx 18^{\circ}$ defector setting and a $30-\mathrm{GeV}$ beam with $\gamma \mathcal{E}_{y} \approx 5 \mu \mathrm{m}$. The deflector-induced beam size on the screen is nearly ten-times larger than nominal, for an rf power input of just $6.5 \mathrm{MW}$ into a $2.4-\mathrm{m}$ structure. Figure 4 shows the 'pitched' beam ( $y$ vs. $z$ ) at $10 \mathrm{MV}$ (left) and $-10 \mathrm{MV}$ (right), by flipping the rf phase. This 2-phase flip technique can be used to separate beam 'pitch' generated upstream of the deflector, caused, for example, by transverse wakefields. The mean beam size for the two phases is then used in Eq. (9). In this case, there is plenty of voltage to examine beams with higher energies and larger emittance values (see Table 1).

With the long bunch, care should be taken that the transverse wakefields generated between deflector and screen do not greatly bias the bunch length measurement results. The wakes are included in the simulation shown here, and are not a significant problem at $1 \mathrm{nC}$ $\left(6.2 \times 10^{9} \mathrm{ppb}\right)$. At a much higher bunch charge the centroid offset might best be removed $(\varphi \approx 0)$ and a perpendicular (horizontal) pulsed steering magnet used for off-axis screen deflection. Similarly, a correlated energy spread can affect the results if $\varphi \neq 0$. A $0.6 \%$ correlated energy spread is included in the simulations.
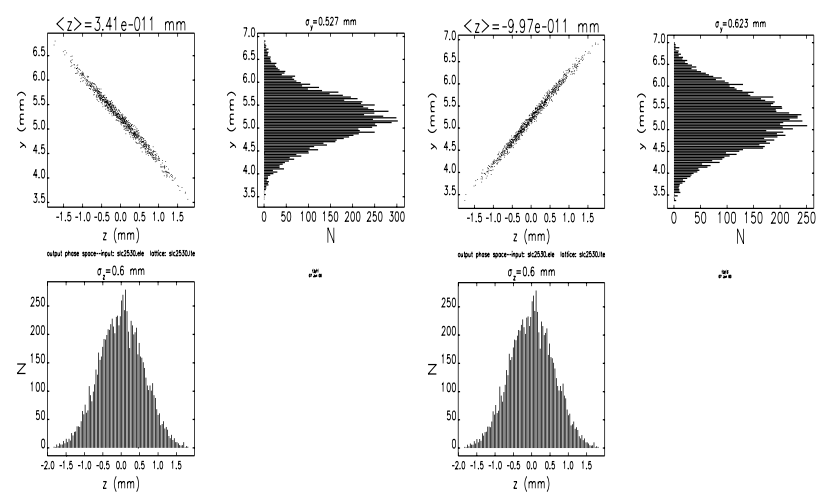

Figure 4 . Vertical vs. longitudinal space at $\varphi=18^{\circ}$ (left 3 plots) and $\varphi=\pi-18^{\circ}$ (right 3 plots). The rms beam size is $527 \mu \mathrm{m}$ (left) and $623 \mu \mathrm{m}$ (right) with asymmetry due to wakes of $1 \mathrm{nC}$.

It may also be interesting to use the existing vertical collimator at the '29-903' location (downstream of the deflector) to 'cut' the vertical spot size and therefore 'cutdown' the bunch length. Of course, the vertical pitch (i.e., the $y-z$ correlation) will still be intact after the cut.

A more complete description of the fields, $2^{\text {nd }}$-order effects, and applications is discussed in reference [9].

\section{REFERENCES}

[1] LCLS Design Study Report, SLAC-R-521, (1998).

[2] "Zeroth-order Design Report for the Next Linear Collider", SLAC-REP-474, May 1996.

[3] G. A. Loew, O. H. Altenmueller, "Design and Applications of R.F. Deflecting Structures at SLAC", PUB-135, Aug. 1965.

[4] R.H. Miller, R.F. Koontz and D.D. Tsang, "The SLAC Injector", IEEE Trans. Nucl. Sci., June 1965, p. 804-8.

[5] X.-J. Wang, "Producing and Measuring Small Electron Bunches", Proc. of the 1999 Part. Acc. Conf., New York, NY, March 1999.

[6] O. H. Altenmueller, et. al., "Investigations of Traveling-Wave Separators for the Stanford TwoMile Linear Accelerator", The Review of Scientific Instruments, Vol. 35, Number 4, April 1964.

[7] G. A. Loew, private communication.

[8] H. Hahn and H. J. Halama, "Design of the Deflector for the R.F. Beam Separator at the Brookhaven AGS", BNL-9306, 1965.

[9] P. Emma et. al., LCLS-TN-00-12, Aug. 2000. 